\title{
Thin current sheets with strong bell-shape guide field: Cluster observations and models with beams
}

\author{
I. Y. Vasko, A. V. Artemyev, A. A. Petrukovich, and H. V. Malova \\ Space Research Institute of Russian Academy of Sciences, Moscow, Russia \\ Correspondence to: I. Y. Vasko (vaskoiy@yandex.ru)
}

Received: 14 July 2014 - Revised: 21 September 2014 - Accepted: 22 September 2014 - Published: 31 October 2014

\begin{abstract}
We study the kinetic structure of intense ion-scale current sheets with strong electron currents and the guide field having a bell-shape profile. We consider four crossings of the Earth magnetotail current sheet by the Cluster mission in 2003. The thickness of these current sheets is about the ion inertial length and significantly smaller than the characteristic ion gyroradius. We analyze the asymmetry of the electron velocity distribution functions and show that the electron current is provided by the small electron subpopulation interpreted as an electron beam or two counter-streaming electron beams. The beam (counter-streaming beams) has a bulk velocity of the order of the electron thermal velocity and a density (difference of beam densities) of about $1-5 \%$ of the plasma density. To describe the observed current sheets we develop a kinetic model with particle beams. The model predicts different thickness of the current sheet for different types of current carriers (one electron beam or two counterstreaming electron beams). The observed ion-scale current sheets can be explained assuming that the current is carried by one electron beam and a co-streaming ion beam. Although the ion beam does not carry a significant current, this beam is required to balance the electron current perpendicular to the current sheet neutral plane. The developed model explains the dominance of the electron current and the ion scales of the current sheets.
\end{abstract}

Keywords. Magnetospheric physics (magnetospheric configuration and dynamics; magnetotail; plasma sheet)

\section{Introduction}

The current sheet (CS) is a critical element of the Earth magnetotail (Ness, 1965, 1969), where charged particle acceleration and magnetic field energy dissipation occur (Birn et al., 2012). Moreover, the CS dynamics determines the evolution of the magnetotail configuration (see, e.g. Sergeev et al., 1993, 2012; Baker et al., 1996). The local structure of the magnetic field in the CS can be usually described as $\boldsymbol{B}=B_{0} \tanh (z / L) \boldsymbol{e}_{x}+B_{y} \boldsymbol{e}_{y}+B_{z} \boldsymbol{e}_{z}$ (the GSM coordinate system is used), where $L$ is the CS thickness, $B_{0}$ is the magnetic field at the CS boundary, shear (or guide) $B_{y}$ and perpendicular $B_{z}$ components are generally uniform across the CS and significantly smaller than $B_{0}$.

The Cluster mission has substantially advanced the understanding of the CS structure (Runov et al., 2006; Artemyev and Zelenyi, 2013) and dynamics (Nakamura et al., 2006; Baumjohann et al., 2007). Asano et al. (2005) and Runov et al. (2006) have shown that the CS structure often cannot be described by the Harris model (Harris, 1962). On contrary the CS is embedded into the thick plasma sheet (McComas et al., 1986; Petrukovich et al., 2011). The CS thickness is often about several ion thermal gyroradii (Runov et al., 2006). The current is frequently provided by electrons (Israelevich et al., 2008; Artemyev et al., 2009).

The results obtained by the Cluster mission have stimulated the development of kinetic CS models (see review by Artemyev and Zelenyi, 2013). Sitnov et al. (2000) and Zelenyi et al. (2000) have developed a 1-D thin CS (TCS) model assuming that the current is provided by transient (or Speiser) ions (Speiser, 1965). In contrast to 2-D models with an isotropic pressure tensor (Birn et al., 2004; Yoon and Lui, 2005; Nickeler and Wiegelmann, 2010; Vasko et al., 2013 ) in the TCS model the magnetic field tension along the $x$ axis is balanced by the ion pressure anisotropy (Eastwood, 1972, 1974). Detailed comparisons with spacecraft observations have shown that the TCS model well describes the CS structure (Artemyev and Zelenyi, 2013). The observed dominance of the electron current (Israelevich et al., 2008; Artemyev et al., 2009) is explained by the Hall effect caused by the earthward electrostatic field (Zelenyi et al., 2010). The 
corresponding cross-field drift decreases the ion current and increases the electron one.

Until recently, observations have been focused on CSs with a small shear component $B_{y}$. On the other hand, Cluster data have shown the presence of CSs with a strong shear component ( $B_{y} \sim B_{0}$ ) having uniform (Shen et al., 2008a; Rong et al., 2012) or bell-shape (Nakamura et al., 2008; Rong et al., 2012; Artemyev et al., 2013; Grigorenko et al., 2013) profiles. Due to the strong $B_{y}$ the current has a significant field-aligned component. For CSs analyzed by Shen et al. (2008a), Nakamura et al. (2008), and Artemyev et al. (2013) the current is generally provided by electrons. The particular feature of CSs reported by Nakamura et al. (2008) and Artemyev et al. (2013) is the CS thickness of about several tenths of the characteristic ion gyroradius.

Artemyev (2011) has generalized the TCS model to take into account the strong bell-shape $B_{y}$. The model assumes that ion beams are the main current carriers and predicts a CS thickness of about several ion thermal gyroradii. Thus, the TCS model is incapable of describing the CSs reported by Nakamura et al. (2008) and Artemyev et al. (2013). First, the model does not explain the dominance of the electron current. In contrast to CSs with small $B_{y}$ the current in CSs with strong $B_{y}$ is unlikely due to the Hall effect, since the current is generally field-aligned. Second, the thickness of the observed CSs is by an order of magnitude smaller than predicted by the TCS model. Thus, these CSs differ from CSs with small $B_{y}$ having ions for the main current carriers and a thickness of about several ion thermal gyroradii.

The present paper aims at the understanding of the kinetic structure of CSs reported by Nakamura et al. (2008) and Artemyev et al. (2013). We consider two questions. (1) What is the origin of the field-aligned electron current from the kinetic point of view? (2) What determines the spatial scale of the CS? We analyze electron distribution functions to address the first question, and develop an analytical model to address the second question. We note that CSs analyzed by Nakamura et al. (2008) and Artemyev et al. (2013) have been observed in 2003, when the Cluster spacecraft separation was suitable for observation of CSs with a thickness of about $300 \mathrm{~km}$. These CSs represent only a subclass of CSs with strong $B_{y}$. The separate study is required for thicker CSs observed by Rong et al. (2012) and Grigorenko et al. (2013) in other periods of the Cluster mission.

The paper is organized as follows. In Sect. 2 we analyze the structure of electron distribution functions. In Sect. 3 we develop the analytical kinetic model and compare it with the Artemyev (2011) model. In Sect. 4 we compare Cluster observations with our model. In Sect. 5 we summarize and discuss our results.

\section{Observations}

We consider four CSs (referred to as CSs nos. 1-4) observed by the Cluster mission in 2003 on the following dates: 24 August (18:43:00-18:43:15), 27 August (18:53:1518:54:10), 19 September (22:38-22:39) and 24 September (14:21-14:24). The detailed analysis of CSs nos. 1-4 can be found in the paper by Artemyev et al. (2013) (called further paper AA2013). Before analyzing electron distribution functions we present an overview of CS no. 4. The following data from the Cluster Active Archive (http://caa.estec. esa.int/caa/) are used: FGM (fluxgate magnetometer) magnetic field with $5 \mathrm{~Hz}$ time resolution (Balogh et al., 2001), CIS (Cluster ion spectrometry)/CODIF (composition and distribution function) proton moments (Rème et al., 2001), PEACE (Plasma Electron and Current Experiment) electron moments (Johnstone et al., 1997). We use measurements of the PEACE-HEEA (high energy electron analyser) sensor to obtain electron moments and distribution functions. We have also checked that PEACE-LEEA (low energy electron analyser) sensor give similar values for electron bulk velocities in all investigated CSs. Electron moments (density $n_{\mathrm{e}}$, temperature $T_{\mathrm{e}}$, bulk velocity $\boldsymbol{v}_{\mathrm{e}}$ ) and electron distribution functions are provided by $\mathrm{C} 2$, while ion moments (temperature $T_{\mathrm{i}}$, bulk velocity $\boldsymbol{v}_{\mathrm{i}}$ ) are provided by $\mathrm{C} 1$. We assume that the electron density is equal to the plasma density.

\subsection{Overview of CS no. 4}

Figure 1a-c present magnetic field profiles for CS no. 4. The shear component $B_{y}$ is $\sim 30 \mathrm{nT}$ so that $B_{y}$ is unlikely due to the IMF (interplanetary magnetic field) (Fairfield, 1979; Petrukovich, 2011). The particular feature presented in Fig. 1d is a weak variation of the magnetic field magnitude $|\boldsymbol{B}|=\left(B_{x}^{2}+B_{y}^{2}+B_{z}^{2}\right)^{1 / 2}$ across the CS. The magnetic field magnitude is within the range from 26 to $32 \mathrm{nT}$. The plasma pressure $p_{0}=n_{\mathrm{e}}\left(T_{\mathrm{e}}+T_{\mathrm{i}}\right)$ shown in Fig. 1e is within the range from 0.2 to $0.3 \mathrm{nPa}$, i.e. $p_{0}$ varies weakly as well. The magnetic field in magnetotail lobes $B_{\text {ext }}^{2}=\boldsymbol{B}^{2}+8 \pi p_{0}$ is generally constant in agreement with the vertical pressure balance across the CS. On average $B_{\text {ext }}$ is about $38 \mathrm{nT}$. Panels d and e indicate that the observed CS is almost force-free suggesting the presence of a significant field-aligned current. Figure if presents the current density determined via the curlometer technique (Chanteur, 2000), while Fig. 1g presents fieldaligned $j_{\|}=\boldsymbol{j} \boldsymbol{B} /|\boldsymbol{B}|$ and perpendicular $j_{\perp}=\left|\boldsymbol{j}-j_{\|} \boldsymbol{B} /\right| \boldsymbol{B} \|$ components. The field-aligned component does actually exceed the perpendicular component by a factor of 5 .

The study of the CS in the local coordinate system $(\boldsymbol{l}, \boldsymbol{m}, \boldsymbol{n})$ allows us to find out whether the CS can be locally considered as a 1-D structure. We determine $(\boldsymbol{l}, \boldsymbol{m}, \boldsymbol{n})$ following Runov et al. (2006): vector $\boldsymbol{l}$ is along the maximum variance direction determined by the MVA (minimum variance analysis) method (Sonnerup and Cahill, 1968); vector $\boldsymbol{m}$ is along the average current density direction and is 

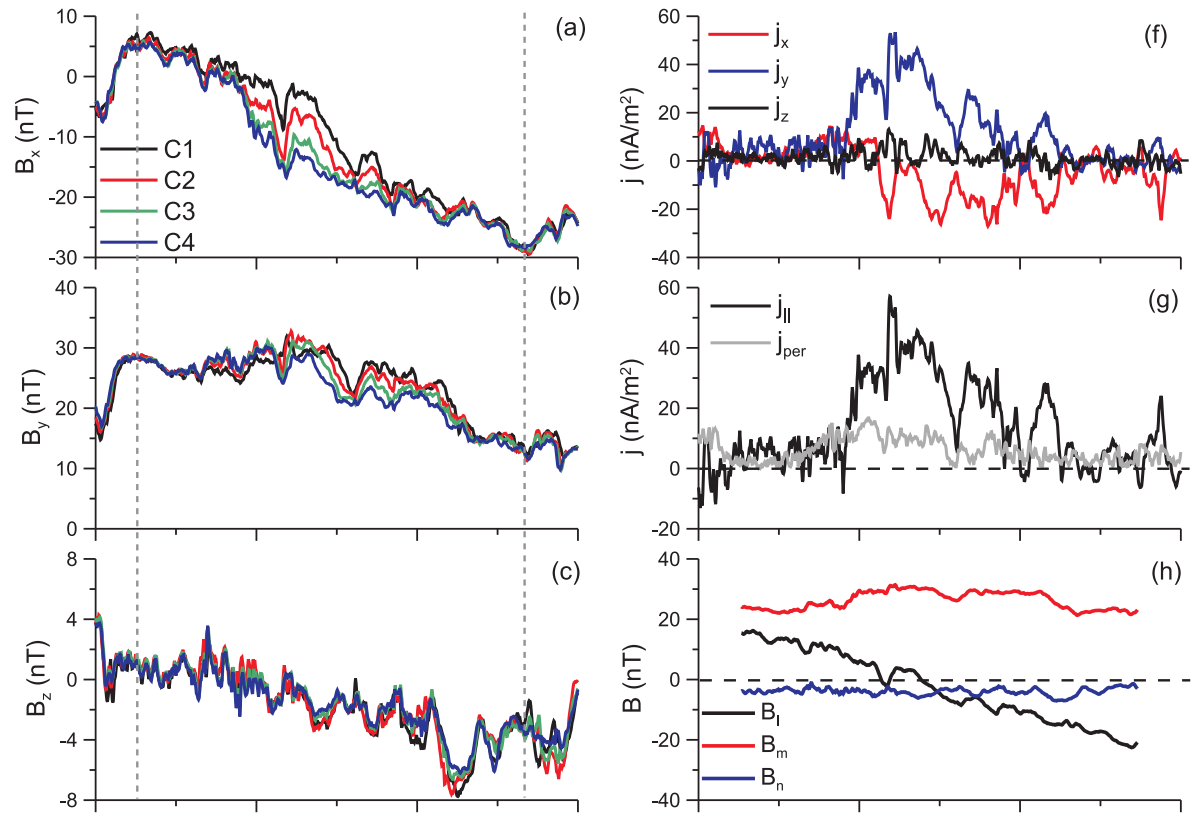

f)
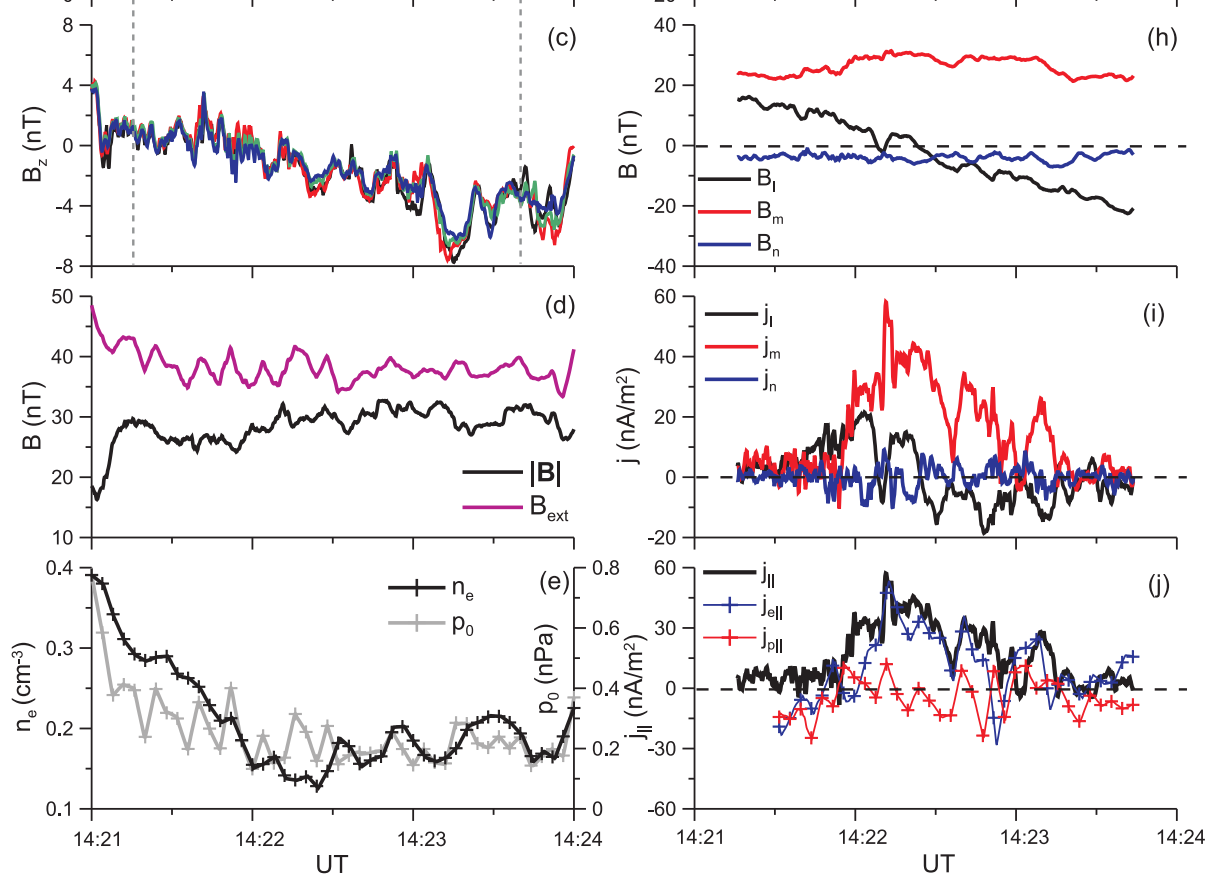

Figure 1. CS observed by the Cluster on 24 September 2003: (a), (b), (c) magnetic field components observed by four Cluster spacecraft; dashed lines mark the interval used to determine the local coordinate system $(\boldsymbol{l}, \boldsymbol{m}, \boldsymbol{n}) ;(\mathbf{d})$ the magnetic field magnitude in the barycenter $|\boldsymbol{B}|$ and in the magnetotail lobes $B_{\text {ext }}$; (e) the electron density and the total plasma pressure $p_{0}=n_{\mathrm{e}}\left(T_{\mathrm{e}}+T_{\mathrm{i}}\right)$; (f) the current density determined using the curlometer technique; (g) current density components parallel and perpendicular to the magnetic field; (h, i) magnetic field and current density in the local coordinate system $(\boldsymbol{l}, \boldsymbol{m}, \boldsymbol{n}) ;(\mathbf{j})$ field-aligned components of the curlometer, ion and electron current densities.

perpendicular to vector $\boldsymbol{l}$; vector $\boldsymbol{n}=\boldsymbol{l} \times \boldsymbol{m}$ completes the right-handed system. We use the data within the interval indicated in Fig. 1a-c and find that $\boldsymbol{l}=[0.91,0.37,0.14], \boldsymbol{m}=$ $[-0.38,0.91,0.1]$, and $\boldsymbol{n}=[-0.1,-0.15,0.98]$. Figure $1 \mathrm{~h}, \mathrm{i}$ present the magnetic field in the Cluster barycenter and the curlometer current density in the local coordinate system. The magnetic field $B_{l}$ has the classical profile with a value $B_{0} \sim 20 \mathrm{nT}$ at the CS boundary. The shear component $B_{m}$ has the bell-shape profile being $\sim 22 \mathrm{nT}$ at the CS boundary and $\sim 32 \mathrm{nT}$ in the neutral plane $\left(B_{l} \approx 0\right)$. The magnetic field $B_{n}$ is $\sim 4 \mathrm{nT}$ and uniform across the CS. There are two major current density components $j_{l}$ and $j_{m}$ having asymmetric and symmetric profiles relative to the neutral plane, respectively. The current density $j_{n}$ is significantly smaller than $j_{l}$ and $j_{m}$.
Figure 1h, i show that CS no. 4 can be considered as a 1-D structure with variations of $B_{l}$ and $B_{m}$ along vector $\boldsymbol{n}$. Figure $1 \mathrm{j}$ presents the field-aligned components of curlometer, ion and electron current densities. The latter are determined as $e n_{\mathrm{e}} \boldsymbol{v}_{\mathrm{i}}$ and $-e n_{\mathrm{e}} \boldsymbol{v}_{\mathrm{e}}$, respectively. The curlometer current is generally described by the electron current.

Thus, CS no. 4 is an almost force-free electron-dominated 1-D CS. CSs nos. 1-3 have similar properties being however partially force-free (see paper AA2013). The thickness of CSs nos. 1-4 is about $300 \mathrm{~km}$, that is only about one tenth of the characteristic ion gyroradius. The characteristic plasma density for these CSs is $\sim 0.15 \mathrm{~cm}^{-3}$ so that the CS thickness is about the ion inertial length (see paper AA2013). 


\subsection{Electron distribution functions}

In CSs with small $B_{y}$ the current is in the dawn-dusk direction and is generally perpendicular to the magnetic field (Runov et al., 2006). Theory predicts that the electron current is due to the drift motion of the whole electron population, so that the electron distribution function $f_{\mathrm{e}}(\boldsymbol{v})$ is shifted along the $v_{y}$ axis. The distribution function remains symmetric with respect to the drift velocity, $v_{y}=v_{D}$. Given that the drift velocity is significantly smaller than the thermal velocity, $v_{\mathrm{Te}} \sim 16000 \mathrm{~T}_{\mathrm{e}}^{1 / 2} \mathrm{~km} \mathrm{~s}^{-1}\left(T_{\mathrm{e}}\right.$ is in $\left.\mathrm{keV}\right)$, the distribution function should be practically symmetric even with respect to $v_{y}=0$.

There are two scenarios for the formation of almost field-aligned electron current in CSs with strong $B_{y}$. In analogy to CSs with small $B_{y}$ the current may be provided by the field-aligned flow of the whole electron population, so that the distribution function remains symmetric with respect to the bulk velocity. On the other hand, the current may be provided by a small subpopulation of electrons (i.e. electron beam/beams). Then, the distribution function becomes in principle asymmetric. To determined the asymmetry of the distribution function with respect to $v_{x}=0$ and $v_{y}=0$ one can consider phase densities $D\left(v_{x}\right)=\int\left(f_{\mathrm{e}}\left(v_{x}, v_{y}, v_{z}\right)-f_{\mathrm{e}}\left(-v_{x}, v_{y}, v_{z}\right)\right) \mathrm{d} v_{y} \mathrm{~d} v_{z} \quad$ and $D\left(v_{y}\right)=\int\left(f_{\mathrm{e}}\left(v_{x}, v_{y}, v_{z}\right)-f_{\mathrm{e}}\left(v_{x},-v_{y}, v_{z}\right)\right) \mathrm{d} v_{x} \mathrm{~d} v_{z} . \quad$ The regions where phase densities $D\left(v_{x}\right)$ and $D\left(v_{y}\right)$ are positive correspond to the subpopulation providing the current.

Figure 2 presents the analysis of electron distribution functions. Four columns correspond to CSs nos. 1-4. The two upper rows present magnetic field and curlometer current density profiles. First, we consider the electron distribution functions in CS no. 4 (the last column). The distribution functions have been measured in points 1,2 and 3 indicated in the upper panel. Point 2 is located in the neutral plane (where $j_{y}$ has maximum), while points 1 and 3 are located farther from the neutral plane (where $j_{x} \neq 0$ ). Therefore we analyze the distribution function $\int f_{\mathrm{e}}(\boldsymbol{v}) \mathrm{d} v_{x} \mathrm{~d} v_{z}$ in point 2 and the distribution functions $\int f_{\mathrm{e}}(\boldsymbol{v}) \mathrm{d} v_{y} \mathrm{~d} v_{z}$ in points 1 and 3 . The three lowest panels present the corresponding distribution functions. The blue regions corresponding to positive phase densities $D\left(v_{x}\right)$ and $D\left(v_{y}\right)$ show that the electron distribution function is asymmetric with respect to $v_{x}=0$ as well as $v_{y}=0$.

The regions with positive phase densities $D\left(v_{x}\right)$ and $D\left(v_{y}\right)$ may correspond to an electron beam streaming along the magnetic field with a bulk velocity of the order of the thermal velocity $v_{\mathrm{Te}}$. The beam density is proportional to the area of the blue filled region. An estimate of this area shows that the beam density is about $5 \%$ of the plasma density. Alternatively, the beam density can be estimated as $\sim j_{\|} / e v_{\mathrm{Te}}$, i.e. we have taken into account that the beam provides most of the current. In CS no. 4 we have $j_{\|} \sim 40 \mathrm{nA} \mathrm{m}^{-2}, T_{\mathrm{e}} \sim$ $2 \mathrm{keV}$ and $n_{\mathrm{e}} \sim 0.15 \mathrm{~cm}^{-3}$ resulting in a beam density of $\sim 0.01 \mathrm{~cm}^{-3}$ or equivalently about $6 \%$ of the plasma density in reasonable accordance with the previous estimate.

The regions with the positive phase densities may also correspond to two counter-streaming electron beams. In this scenario the regions with positive phase densities arise due to different densities of the beams. The bulk velocities of the beams should be approximately equal, since otherwise the region with positive phase densities $D\left(v_{x}\right)$ and $D\left(v_{y}\right)$ would be observed at both sides with respect to $v_{x}=0$ and $v_{y}=0$.

Figure 2 also presents the analysis of the asymmetry of the electron distribution function in CSs nos. 1-3. The first column presents the distribution functions over $v_{x}$ and $v_{y}$, i.e. $\int f_{\mathrm{e}}(\boldsymbol{v}) \mathrm{d} v_{y} \mathrm{~d} v_{z}$ and $\int f_{\mathrm{e}}(\boldsymbol{v}) \mathrm{d} v_{x} \mathrm{~d} v_{z}$, observed at some distance from the neutral plane in CS no. 1. The regions with positive phase densities $D\left(v_{x}\right)$ and $D\left(v_{y}\right)$ can be seen. The second column presents the distribution function over $v_{y}$ observed in CS no. 2 near the neutral plane and the distribution functions over $v_{x}$ observed farther from the neutral plane. The regions of positive phase densities $D\left(v_{x}\right)$ and $D\left(v_{y}\right)$ are present again. The third column shows that there is a small asymmetry of the electron distribution function in CS no. 3 . Thus, the distribution functions in CSs nos. 1-3 have the asymmetry similar to the one observed in CS no. 4. The current density profiles presented in Fig. 2, and the electron temperatures and densities presented in Table 2 in paper AA2013, allow to estimate the beam density (difference of beam densities). We have found that in CSs nos. 1-3 the beam density (difference of beam densities) is about 5.3, 3.7 and $1.3 \%$ of the plasma density, respectively.

Thus, the current in CSs nos. 1-4 is provided by a small subpopulation of electrons. The present analysis does not show whether this subpopulation is formed by one electron beam or two counter-streaming electron beams with approximately equal bulk velocities. In the next section we develop the simple analytical CS model demonstrating that the CS thickness turns out to be different for these alternative scenarios.

\section{The analytical model}

We consider a magnetoplasma structure with the magnetic field

$\boldsymbol{B}=B_{0} \sin (k z) \boldsymbol{e}_{x}+B_{0} \cos (k z) \boldsymbol{e}_{y}+B_{z} \boldsymbol{e}_{z}$.

Kinetic models of such structures have been developed to describe large-amplitude circularly polarized electromagnetic waves in magnetized plasma (Bell, 1965; Lutomirski and Sudan, 1966; Sonnerup and Su, 1967; Vasko et al., 2014). The half period $k z \in[-\pi / 2, \pi / 2]$ of this structure shown in Fig. 3 represents a force-free 1-D CS with a bell-shape $B_{y}$. Self-consistent kinetic models allow to determine the CS thickness $L=k^{-1}$ for specified particle distribution functions. 

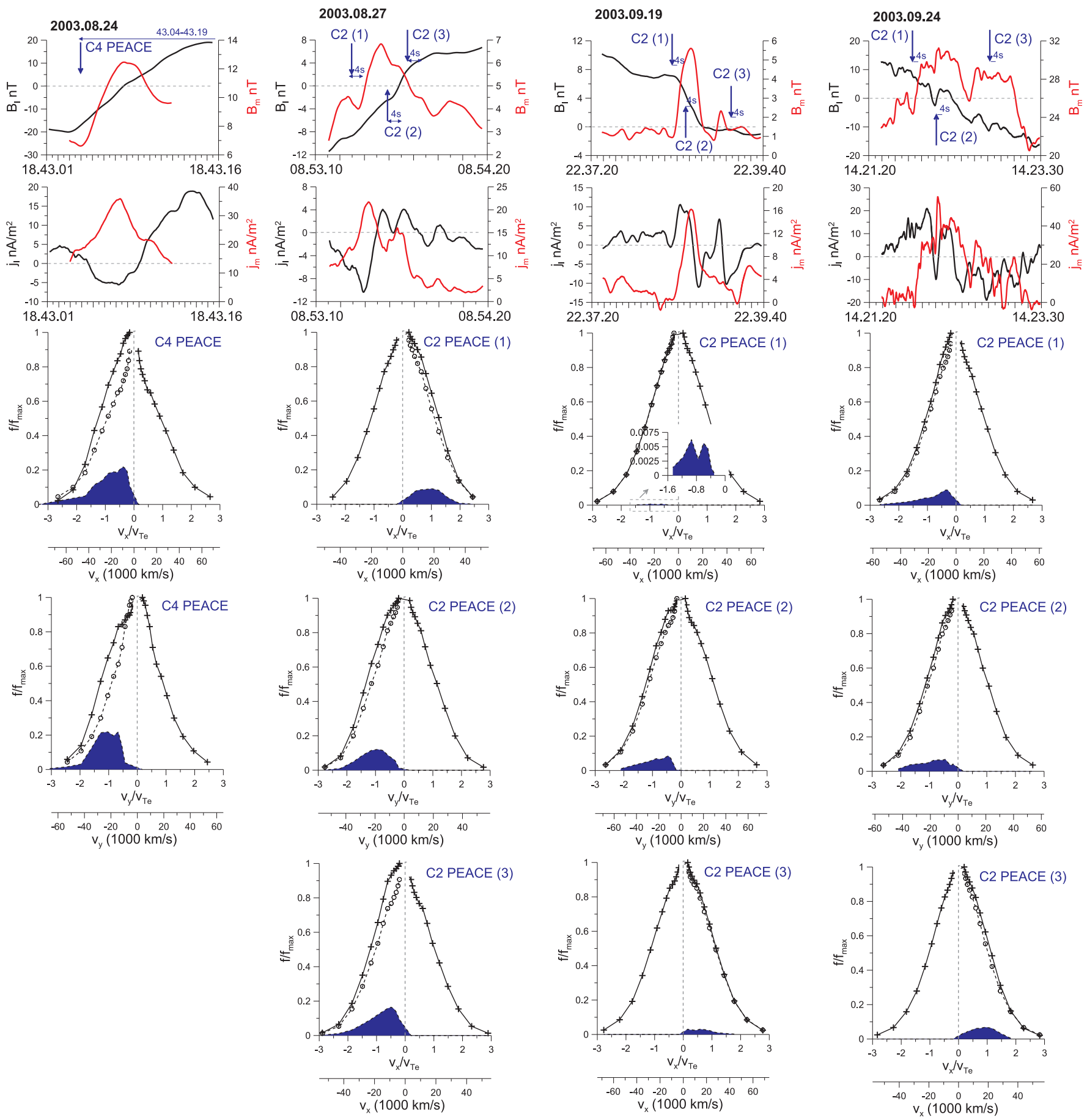

Figure 2. The analysis of electron distribution functions observed in CSs nos. 1-4. Four columns correspond to CSs nos. 1-4, respectively. The first and second rows show profiles of $\boldsymbol{l}$ and $\boldsymbol{m}$-components of the magnetic field and the curlometer current density. The lower rows present electron distribution functions $\int f_{\mathrm{e}}(\boldsymbol{v}) \mathrm{d} v_{x} \mathrm{~d} v_{z}$ or $\int f_{\mathrm{e}}(\boldsymbol{v}) \mathrm{d} v_{y} \mathrm{~d} v_{z}$ at points indicated with arrows in the first row. In each panel the true distribution function normalized over its maximum value is shown by crosses. Circles present the reflection of one of the wings of the distribution function with respect to $v_{y}=0$ or $v_{x}=0$. The difference of the true distribution function and the reflected part is indicated by the blue filled region. 


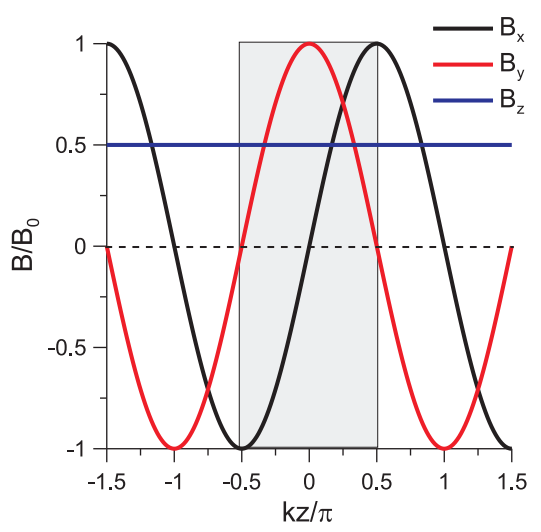

Figure 3. The magnetic field (1), where for the illustration we have assumed $B_{z} / B_{0}=0.5$. The half period corresponding to the CS with bell-shape $B_{y}$ is marked.

In the absence of electrostatic fields the particle dynamics in the magnetic field (1) can be described using three integrals of motion. We assume that there is no electrostatic field in a rest frame and show in Appendix that the exact charge neutrality may be actually satisfied. The adequacy of this assumption from the point of view of observations is discussed in Sect. 5. The integrals of motion are the particle energy $W_{\alpha}$, the generalized momentum $p_{y, \alpha}$ and the helical integral $I_{\alpha}($ Bell, 1965) conserved due to the helical symmetry of the magnetic field (1):

$$
\begin{aligned}
& W_{\alpha}=\boldsymbol{v}_{\perp}^{2}+v_{z}^{2} \\
& p_{y, \alpha}=v_{y}+\frac{q_{\alpha} B_{y}}{m_{\alpha} k}+\Omega_{\alpha} x \\
& I_{\alpha}=2 \frac{\Omega_{\alpha}}{k} v_{z}+\left(\boldsymbol{v}_{\perp}+\frac{q_{\alpha} \boldsymbol{B}_{\perp}}{m_{\alpha} k}\right)^{2},
\end{aligned}
$$

where $\alpha=i, e$ corresponds to ions and electrons, $q_{\alpha}, m_{\alpha}$ are particle charges and masses $\left(q_{\mathrm{i}}=-q_{\mathrm{e}} \equiv e\right), \Omega_{\alpha}=q_{\alpha} B_{z} / m_{\alpha}$ is the particle gyrofrequency, $\boldsymbol{v}_{\perp}=v_{x} \boldsymbol{e}_{x}+v_{y} \boldsymbol{e}_{y}$ and $\boldsymbol{B}_{\perp}=$ $B_{x} \boldsymbol{e}_{x}+B_{y} \boldsymbol{e}_{y}$. To develop a stationary model, the particle distribution functions are chosen as functions of the integrals of motion. Since $p_{y, \alpha}$ depends on the coordinate $x$ the distribution functions for 1-D CS should be chosen as $f_{\alpha}=f_{\alpha}\left(W_{\alpha}, I_{\alpha}\right)$. In the Appendix we show that distribution functions of this general form allow developing a selfconsistent model of the magnetoplasma structure with the magnetic field (1). The distribution functions $f_{\alpha}$ should satisfy only one condition:

$$
\sum_{\alpha=i, e} q_{\alpha} \int v_{z} f_{\alpha} d^{3} \boldsymbol{v}=0
$$

implying the absence of the current along the $z$ axis in accordance with the magnetic field configuration (1).

The particular choice of particle distribution functions allows to develop models of electron- and ion-dominated CSs.
We develop these models and obtain estimates of the CS thickness choosing particle distribution functions in the simplest form.

\subsection{Electron-scale electron-dominated CSs}

Let us assume that the current is carried by electrons. The ion distribution function is chosen as $f_{\mathrm{i}}=f_{\mathrm{i}}\left(W_{\mathrm{i}}\right)$. The electron distribution function $f_{\mathrm{e}}=f_{\mathrm{e}}\left(W_{\mathrm{e}}, I_{\mathrm{e}}\right)$ is an arbitrary function. For illustration we choose $f_{\mathrm{e}}$ in the simplest form:

$f_{\mathrm{e}}=C_{+} \exp \left(-\frac{s_{+} I_{\mathrm{e}}+W_{\mathrm{e}}}{2 u_{+}^{2}}\right)+C_{-} \exp \left(-\frac{s_{-} I_{\mathrm{e}}+W_{\mathrm{e}}}{2 u_{-}^{2}}\right)$,

where $s_{ \pm}, C_{ \pm}, u_{ \pm}$are constant parameters. This distribution function can be written as a sum $f=f_{+}+f_{-}$of distribution functions of two Maxwellian beams:

$$
f_{ \pm}=\frac{n_{ \pm}\left(1+s_{ \pm}\right)}{\left(2 \pi u_{ \pm}^{2}\right)^{3 / 2}} \exp \left[-\frac{\left(v_{z}-v_{z \pm}\right)^{2}}{2 u_{ \pm}^{2}}-\frac{\left(\boldsymbol{v}_{\perp}-\boldsymbol{v}_{ \pm}\right)^{2}}{2 u_{ \pm}^{2}\left(1+s_{ \pm}\right)^{-1}}\right],
$$

where $n_{ \pm}, u_{ \pm}$are beam densities and thermal velocities, while the bulk velocity is determined as

$v_{z \pm}=s_{ \pm} \frac{e B_{z}}{m_{\mathrm{e}} k}, \quad \mathbf{v}_{ \pm}=\frac{s_{ \pm}}{1+s_{ \pm}} \frac{e \boldsymbol{B}_{\perp}}{m_{\mathrm{e}} k}$.

The constant parameters $s_{ \pm}$satisfy the following inequality $s_{ \pm}>-1$, since otherwise the distribution function does not decrease at infinity. We assume that $s_{+}$is positive so that $f_{+}$describes the beam streaming from the southern lobe to the northern lobe. On the other hand, $f_{-}$describes the counter-streaming beam required to balance the current along the $z$ axis, i.e. to satisfy condition (2). This condition implies that $n_{+} v_{z+}+n_{-} v_{z-}=0$ and results in the following relation:

$n_{-} s_{-}+n_{+} s_{+}=0$.

The net current density provided by both beams is

$\boldsymbol{j}_{\perp}=-\left(\frac{n_{+} s_{+}}{1+s_{+}}+\frac{n_{-} s_{-}}{1+s_{-}}\right) \frac{e^{2} \boldsymbol{B}_{\perp}}{m_{\mathrm{e}} k}$.

Using Eq. (4) and beam velocities $v_{ \pm}=\left|\boldsymbol{v}_{ \pm}\right|$determined by Eq. (3) we exclude parameters $s_{ \pm}$from the latter expression and obtain

$\boldsymbol{j}_{\perp}=\frac{\omega_{\mathrm{b}, \mathrm{e}}^{2} \boldsymbol{B}_{\perp}}{k} \frac{\left(n_{+} v_{+}-n_{-} v_{-}\right)^{2}}{n_{\mathrm{b}}^{2} v_{+} v_{-}}$,

where $n_{\mathrm{b}}=n_{+}+n_{-}$is the density of both beams, $\omega_{\mathrm{b}, \mathrm{e}}=$ $\left(n_{\mathrm{b}} e^{2} / m_{\mathrm{e}} \varepsilon_{0}\right)^{1 / 2}$ is the beam plasma frequency $\left(\varepsilon_{0}\right.$ is the dielectric permittivity of vacuo). Finally, the Ampere equation rot $\boldsymbol{B}_{\perp}=\mu_{0} \boldsymbol{j}_{\perp}$ ( $\mu_{0}$ is the permeability of vacuo) allows to determine the CS thickness:

$L \equiv k^{-1}=\frac{c}{\omega_{\mathrm{b}, \mathrm{e}}} \frac{n_{\mathrm{b}}\left(v_{+} v_{-}\right)^{1 / 2}}{\left|n_{+} v_{+}-n_{-} v_{-}\right|}$, 
where $c=\left(\varepsilon_{0} \mu_{0}\right)^{-1 / 2}$ is the speed of light. Thus, the CS thickness scales as the inertial length of electron beams and depends on the bulk velocities of the beams. The estimate of the CS thickness can be also expressed in terms of characteristic electron gyroradii, $\rho_{ \pm}=v_{ \pm} m_{\mathrm{e}} / e B_{0}$ (note that this electron gyroradius depends on the electron bulk velocity $v_{ \pm}$ rather than the thermal velocity $u_{ \pm}$and could be called for the sake of brevity a "beam gyroradius"). Equation (3) can be written in the following form:

$\rho_{ \pm}= \pm \frac{s_{ \pm}}{1+s_{ \pm}} L$.

We exclude parameters $s_{ \pm}$using Eq. (4) and obtain the following estimate of the CS thickness:

$L=\frac{\rho_{+} \rho_{-} n_{\mathrm{b}}}{n_{-} \rho_{-}-n_{+} \rho_{+}}$.

Comparing estimates (5) and (6) we find that the electron inertial length can be expressed as $c / \omega_{\mathrm{b}, \mathrm{e}}=\left(\rho_{+} \rho_{-}\right)^{1 / 2}$. Thus, the CS thickness scales as the characteristic electron gyroradius as well. In the particular case of equal bulk velocities of beams, $v_{+} \approx v_{-}, \rho_{+} \approx \rho_{-}$, and different densities, $n_{-}-n_{+}=\Delta n_{\mathrm{b}}$, we obtain

$L=\frac{c}{\omega_{\mathrm{b}, \mathrm{e}}}\left(\frac{n_{\mathrm{b}}}{\Delta n_{\mathrm{b}}}\right)=\rho_{+} \frac{n_{\mathrm{b}}}{\Delta n_{\mathrm{b}}}$.

We can easily take into account the presence of background ions and electrons with distribution functions $f_{\alpha}=$ $f_{\alpha}\left(W_{\alpha}\right)$. The background plasma does not contribute to the current and hence does not influence the estimate of the CS thickness. The density of background populations is uniform.

Denoting the total plasma density (beams plus background plasma) by $n_{\mathrm{e}}$ we write the estimate of the CS thickness in the form

$L=\frac{c}{\omega_{\mathrm{p}, \mathrm{e}}} \frac{\left(n_{\mathrm{b}} n_{\mathrm{e}}\right)^{1 / 2}}{\Delta n_{\mathrm{b}}}$,

where $\omega_{\mathrm{p}, \mathrm{e}}=\left(n_{\mathrm{e}} e^{2} / m_{\mathrm{e}} \epsilon_{0}\right)^{1 / 2}$ is the electron plasma frequency.

\subsection{Ion-scale ion-dominated CSs}

In analogy we develop the model of an ion-scale iondominated CS. The electron distribution function is chosen as $f_{\mathrm{e}}=f_{\mathrm{e}}\left(W_{\mathrm{e}}\right)$. Assuming the ion distribution function $f_{\mathrm{i}}\left(W_{\mathrm{i}}, I_{\mathrm{i}}\right)$ in the from of two counter-streaming Maxwellian beams we obtain an estimate of the CS thickness similar to the previous section (electron parameters should be replaced by ion ones). In particular case, $v_{+} \approx v_{-}$, the CS thickness is determined as $L=\rho_{+}\left(n_{\mathrm{b}} / \Delta n_{\mathrm{b}}\right)$, so that the CS thickness scales as the ion beam gyroradius.

It is instructive to compare this model with the TCS model developed by Artemyev (2011). In the TCS model the magnetic field is $\boldsymbol{B}=B_{x}(z) \boldsymbol{e}_{z}+B_{y}(z) \boldsymbol{e}_{z}+B_{z} \boldsymbol{e}_{z}$, where $B_{x}(-z)=-B_{x}(z), B_{x}$ is uniform at the CS boundary, i.e.
$\left.B_{x}\right|_{z \rightarrow \infty}=B_{0}, B_{y}$ has a bell-shape profile with a constant value at the CS boundary, i.e. $\left.B_{y}\right|_{z \rightarrow \infty}=$ const, and magnetic field lines are assumed to be stretched, $B_{z} \ll B_{0}$. In the TCS model the current density is carried by counter-streaming ion beams. The ion population is assumed to be unmagnetized and the ion dynamics is described using quasi-adiabatic invariant (Büchner and Zelenyi, 1989). The electron population is magnetized and is described in the frame of the MHD (Magnetohydrodynamics) approach.

In contrast to the TCS model the magnetic field in our model is non-uniform at $|k z|>\pi / 2$ (see Fig. 3) and $B_{y}$ is zero at the CS boundary, $|k z|=\pi / 2$. At the same time in both models the current is provided by counter-streaming ion beams. Thus, the TCS model and our model are similar from the kinetic point of view. Moreover, both models predict the CS thickness of the order of the ion beam inertial length or the ion gyroradius. In contrast to the TCS model, in our model $B_{z}$ has an arbitrary value with respect to $B_{0}$ and the electron population is taken into account in the frame of the kinetic approach. Therefore, the developed model complements the TCS model and allows to extend estimates of the CS thickness for the case where the dominant current is carried by the electron population.

\subsection{Ion-scale electron/ion-dominated CSs}

We have assumed heretofore that the current is provided by counter-streaming electron or ion beams. The counterstreaming beam is required to satisfy Eq. (2). Alternatively, Eq. (2) can be satisfied assuming the presence of one electron beam and a co-streaming ion beam. In this subsection ion and electron quantities are denoted by subscripts \pm (e.g. $m_{+}$and $m_{-}$correspond to ion and electron masses). To develop an illustrative model we choose electron and ion distributions as above in the form of Maxwellian beams:

$$
f_{ \pm}=\frac{n_{ \pm}\left(1+s_{ \pm}\right)}{\left(2 \pi u_{ \pm}^{2}\right)^{3 / 2}} \exp \left[-\frac{\left(v_{z}-v_{z \pm}\right)^{2}}{2 u_{ \pm}^{2}}-\frac{\left(\boldsymbol{v}_{\perp}-\boldsymbol{v}_{ \pm}\right)^{2}}{2 u_{ \pm}^{2}\left(1+s_{ \pm}\right)^{-1}}\right],
$$

where bulk velocities are

$v_{z \pm}=\mp s_{ \pm} \frac{e B_{z}}{m_{ \pm} k} \quad \boldsymbol{v}_{ \pm}=\mp \frac{s_{ \pm}}{1+s_{ \pm}} \frac{e \boldsymbol{B}_{\perp}}{m_{ \pm} k}$.

Densities $n_{ \pm}$of ion and electron beams do not necessarily coincide. The exact charge neutrality may be reached by inclusion of background ion and electron populations with appropriate densities (note that densities of background populations with distribution functions $f_{\alpha}=f_{\alpha}\left(W_{\alpha}\right)$ are uniform (see Appendix)).

Equation (2) results in the following relation:

$n_{+} s_{+} / m_{+}+n_{-} s_{-} / m_{-}=0$.

The net current provided by electron and ion beams is

$\boldsymbol{j}_{\perp}=-\frac{e^{2} \boldsymbol{B}_{\perp}}{k} \frac{n_{+} s_{+}}{m_{+}} \frac{s_{-}-s_{+}}{\left(1+s_{+}\right)\left(1+s_{-}\right)}$. 
Using Eq. (8) and the expressions for beam bulk velocities $v_{ \pm}=\left|\boldsymbol{v}_{ \pm}\right|$we exclude parameters $s_{ \pm}$from the latter expression and obtain

$\boldsymbol{j}_{\perp}=-\frac{e^{2} \boldsymbol{B}_{\perp}}{k} \frac{\left(n_{+} v_{+}-n_{-} v_{-}\right)^{2}}{v_{+} v_{-}\left(m_{+} n_{-}+m_{-} n_{+}\right)}$.

In realistic situations we have $m_{+} n_{-}+m_{-} n_{+} \approx m_{+} n_{-}$ and obtain the following estimate of the CS thickness:

$L=\frac{c}{\omega_{\mathrm{b}, \mathrm{i}}} \frac{\left(n_{+} v_{+} n_{-} v_{-}\right)^{1 / 2}}{\left|n_{+} v_{+}-n_{-} v_{-}\right|}$,

where $\omega_{\mathrm{b}, \mathrm{i}}=\left(n_{+} e^{2} / m_{+} \varepsilon_{0}\right)$ is the ion beam inertial length. For electron-dominated CSs we have $n_{-} v_{-} \gg n_{+} v_{+}$and the CS thickness is

$L \approx \frac{c}{\omega_{\mathrm{b}, \mathrm{i}}}\left(\frac{n_{+} v_{+}}{n_{-} v_{-}}\right)^{1 / 2}$,

while for ion-dominated CSs, $n_{+} v_{+} \gg n_{-} v_{-}$, we have

$L \approx \frac{c}{\omega_{\mathrm{b}, \mathrm{i}}}\left(\frac{n_{-} v_{-}}{n_{+} v_{+}}\right)^{1 / 2}$.

In both cases the CS thickness scales as the ion beam inertial length and depends on the relation between electron and ion currents.

Taking into account the presence of the background plasma the estimate of the thickness of the electrondominated CSs can be written as

$L \approx \frac{c}{\omega_{p, i}}\left(\frac{n_{\mathrm{e}}}{n_{+}}\right)^{1 / 2}\left(\frac{n_{+} v_{+}}{n_{-} v_{-}}\right)^{1 / 2}$,

where $\omega_{p, i}=\left(n_{\mathrm{e}} e^{2} / m_{\mathrm{i}} \varepsilon_{0}\right)^{1 / 2}$ is the ion plasma frequency, $n_{\mathrm{e}}$ is the plasma density. The estimate (9) shows that the CS thickness is proportional to the ion inertial length multiplied by two parameters. The first parameter is larger than unity and is determined by the ion beam density. For the beam density of $1-5 \%$ of the plasma density this parameter is within the range 4.5-10. On contrary, the second parameter is small due to the dominance of the electron current. If the electron current is 10-100 times larger than the ion current this parameter is within the range $0.1-0.3$. Thus, for more or less realistic parameters the CS thickness is of the order of the ion inertial length.

\section{Comparison between cluster observations and models}

The asymmetry of the electron distribution function observed in CSs nos. 1-4 has been ascribed either to one or two counter-streaming electron beams. Our model predicts that the CS thickness scales as the electron inertial length in case of two counter-streaming electron beams and as the ion inertial length in the case of one electron beam and the costreaming ion beam. Thus, the comparison between observations and our model helps to understand the kinetic structure of CSs nos. 1-4.
In the presence of two counter-streaming electron beams with coinciding bulk velocities and different densities the CS thickness is determined by Eq. (7). According to Sect. 2.2 we have $\Delta n_{\mathrm{b}} \sim 0.01 n_{\mathrm{e}}$ for CS no. 3 and $\Delta n_{\mathrm{b}} \sim(0.03-0.05) n_{\mathrm{e}}$ for CSs nos. 1, 2, 4. Therefore, for CS no. 3 we have

$L \sim 100\left(n_{\mathrm{b}} / \Delta n_{\mathrm{b}}\right)^{1 / 2} \mathrm{~km}$

while for CSs nos. 1, 2, 4 we have

$L \sim 50\left(n_{\mathrm{b}} / \Delta n_{\mathrm{b}}\right)^{1 / 2} \mathrm{~km}$.

In Sect. 2.2 we have determined the difference of beam densities $\Delta n_{\mathrm{b}}$, while the total density of the beams $n_{\mathrm{b}}$ could not be determined. Observations show that the thickness of CSs nos. 1-4 is about $300 \mathrm{~km}$ (Artemyev et al., 2013), so that our estimates are in agreement with observations if $n_{\mathrm{b}} \sim 10 \Delta n_{\mathrm{b}} \sim 0.1 n_{\mathrm{e}}$ for CS no. 3 and $n_{\mathrm{b}} \sim 30 \Delta n_{\mathrm{b}} \sim n_{\mathrm{e}}$ for CSs nos. 1, 2, 4. Thus, the presence of two electron beams seems to be realistic for CS no. 3 and is quite unrealistic for CSs nos. 1, 2, 4.

Let us assume that there is only one electron beam. To balance the electron current along the $z$ axis one requires the presence of a co-streaming ion beam. It seems reasonable to assume that the ion beam density $n_{+}$is of the order of the electron beam density. Thus, the CS thickness of electrondominated CSs nos. 1-4 is determined by Eq. (9), where according to Sect. 2.2 we have $n_{+} \approx n_{-} \sim(0.01-0.05) n_{\mathrm{e}}$. Then, the thickness of CSs nos. $1-4$ is

$L \sim(4.5-10) \frac{c}{\omega_{p, i}}\left(\frac{n_{+} v_{+}}{n_{-} v_{-}}\right)^{1 / 2}$,

where the ion inertial length for the characteristic density $n_{\mathrm{e}} \sim 0.15 \mathrm{~cm}^{-3}$ in CSs nos. $1-4$ is about $500 \mathrm{~km}$. The ratio of electron and ion currents is not well-defined due to the smallness of the ion current. However, for a quite reasonable ratio of electron and ion current ranging from about 10 to 100 we obtain a CS thickness of the order of the ion inertial length in agreement with the observations.

We conclude that the structure of CS no. 3 can be explained by both scenarios (counter-streaming electron beams or co-streaming electron and ion beams), while the structure of CSs nos. 1, 2, 4 can be better explained assuming the presence of one electron beam and a co-streaming ion beam.

\section{Discussion}

We have analyzed the structure of CSs with strong $B_{y}$ reported earlier by Artemyev et al. (2013). The observation of one similar CS has been presented by Nakamura et al. (2008). The peculiar features of these CSs is the dominance of the electron current and the thickness of about the ion inertial length (but only several tenths of the characteristic ion gyroradius). We have answered both questions posed in the 
Introduction. (1) What is the origin of the field-aligned electron current from the kinetic point of view? (2) What determines the spatial scale of the CS? We have shown that the electron current is provided by the electron beam (or beams for CS no. 3) streaming along the magnetic field with velocities of the order of the thermal velocity and having density (difference of densities) of about 1-5\% of the plasma density. We have developed a simple analytical model demonstrating that a co-streaming ion beam for CSs nos. 1, 2, 4 (or counter-streaming electron beam for CS no. 3 ) should be present. The ion beam is required to balance the electron current perpendicular to the CS neutral plane. The CS thickness predicted by the model is in agreement with observations. We have not analyzed the asymmetry of ion distribution functions to prove experimentally the presence of co-streaming ion beams. In fact, the method of the asymmetry analysis used in the present paper for electrons is not likely appropriate for ions due to smallness of the ion current.

The CSs reported by Nakamura et al. (2008); Artemyev et al. (2013) form only a subclass of CSs with strong $B_{y}$. First, in these CSs the shear component $B_{y}$ has a bell-shape profile (CSs with uniform $B_{y}$ profile have not been discussed in this paper). Second, these CSs are very thin (observed by Cluster in 2003) and hence very intense. Such thin and intense CSs are usually formed in the vicinity of the reconnection region explaining the presence of electron and ion beams reported in the present study. Shen et al. (2008b), Rong et al. (2012), and Grigorenko et al. (2013) have reported observation of CSs with strong uniform/bell-shape $B_{y}$ with larger thickness. The investigation of properties of these CSs requires a separate study. The model developed in the present study can be also useful for such analysis.

The kinetic CS models taking into account the presence of the guide field have been developed in numerous studies (Roth et al., 1996; Harrison and Neukirch, 2009; Panov et al., 2011; De Keyser and Echim, 2013; De Keyser et al., 2013). However, in these models the magnetic field $B_{z}$ is zero, while this component is always present in the Earth magnetotail. There are only few kinetic models describing CSs with strong uniform/bell-shape $B_{y}$ and non zero $B_{z}$. Artemyev (2011) and Mingalev et al. (2012) have developed the TCS model with strong bell-shape $B_{y}$ and small $B_{z}$. The kinetic models of CSs with strong uniform $B_{y}$ have not been developed yet. The development of CS models with strong $B_{y}$ is important not only for the description of CSs in the Earth magnetotail. CSs with strong bell-shape $B_{y}$ are frequently observed in the Jovian magnetotail (Behannon et al., 1981; Artemyev et al., 2014).

In the present paper we have developed a rather simple analytical model having several advantages with respect to the TCS model. First, in this model both electrons and ions are considered in the frame of the kinetic approach. We have considered the cases when the current is carried by counter-streaming electron beams, counter-streaming ion beams, an electron beam and a co-streaming ion beam. In the
TCS model the current is always assumed to be carried by counter-streaming ion beams (Artemyev, 2011). The kinetic description of electron and ion populations is important for our analysis, since the CS thickness can be determined only in the frame of the kinetic approach. Second, in the developed model the $B_{z}$ component can be arbitrary large. On the other hand, the developed model is not sufficiently flexible, since the magnetic field has a specific prescribed configuration (1). Thus, the development of CS models with strong uniform/bell-shape $B_{y}$ with a more flexible magnetic field configuration is required.

In the developed class of models the densities of beams and background populations are uniform across the CS. We have chosen these densities so that the exact charge neutrality is satisfied. As a result the plasma polarization does not occur and the corresponding electrostatic field is absent. In observed CSs the charge neutrality may be satisfied only approximately resulting in the appearance of the electrostatic field directed perpendicular to the CS neutral plane (approximately along the $z$ axis). A sufficiently strong electrostatic field violates the conservation of the helical integral. Thus, our model cannot be straightforwardly generalized to take into account polarization effects as in 1-D models of tangential discontinuities $\left(B_{z}=0\right)$ (Yoon and Lui, 2004; De Keyser and Echim, 2013; De Keyser et al., 2013). The development of 1-D CS models with $B_{z} \neq 0$, the bell-shape $B_{y}$ and taking into account possible polarization effects presents significant interest. At the same time, such models could not be completely verified using currently available experimental data, since electric field $E_{z}$ is not reliably measured by the Cluster spacecraft. Finally, we note that our model being rather simplified describes well the magnetic field distribution and the structure of the electron distribution functions in observed CSs.

\section{Conclusion}

The results of the present study are summarized below

1. In the analyzed CSs the electron distribution function has an asymmetric structure attributed to the presence of an electron beam (two counter-streaming electron beams) with a bulk velocity of the order of the thermal velocity and a density (difference of densities) of about $1-5 \%$ of the plasma density.

2. We have developed an analytical model of the force-free 1-D CS with bell-shape $B_{y}$ and a finite $B_{z}$. The developed model well describes the properties of observed CSs (the observed CS thickness, the dominance of the electron current).

3. In case of one electron beam the model predicts the presence of a co-streaming ion beam required to balance the electron current perpendicular to the CS neutral plane. 


\section{Appendix A:}

Distribution functions $f_{\alpha}\left(W_{\alpha}, I_{\alpha}\right)$ depend on the magnetic field $\boldsymbol{B}_{\perp}$ only in combination $\boldsymbol{v}_{\perp}+q_{\alpha} \boldsymbol{B}_{\perp} / m_{\alpha} k$. Therefore ion and electron densities

$n_{\alpha}=\int f_{\alpha}\left(W_{\alpha}, I_{\alpha}\right) \mathrm{d} v_{z} d^{2} \boldsymbol{v}_{\perp}=$ const

are uniform. Thus, the exact charge neutrality can be satisfied by choosing equal densities of electron and ion populations, i.e. $n_{\mathrm{i}}=n_{\mathrm{e}}$.

Introducing cylindrical coordinates in the velocity space $v_{x}=v_{\perp} \cos \varphi$ and $v_{y}=v_{\perp} \sin \varphi$ and taking into account that $\boldsymbol{B}_{\perp}^{2}=B_{0}^{2}=$ const we write the helical integral in the form $I_{\alpha}=2 \frac{\Omega_{\alpha}}{k} v_{z}+v_{\perp}^{2}+2 v_{\perp} \frac{q_{\alpha} B_{0}}{m_{\alpha} k} \sin (\varphi+k z)+\left(\frac{q_{\alpha} B_{0}}{m_{\alpha} k}\right)^{2}$.

Then the net current densities $j_{x}$ and $j_{y}$ are determined as

$j_{x}=\sum_{\alpha=i, e} q_{\alpha} \int v_{\perp}^{2} \cos (\tilde{\varphi}-k z) f_{\alpha}\left(v_{z}, v_{\perp}, \sin \varphi\right) \mathrm{d} v_{z} \mathrm{~d} v_{\perp} d \tilde{\varphi}$ $j_{y}=\sum_{\alpha=i, e} q_{\alpha} \int v_{\perp}^{2} \sin (\tilde{\varphi}-k z) f_{\alpha}\left(v_{z}, v_{\perp}, \sin \varphi\right) \mathrm{d} v_{z} \mathrm{~d} v_{\perp} d \tilde{\varphi}$,

where $\tilde{\varphi}=\varphi+k z$. We note that for any function $g=g(\sin \tilde{\varphi})$ the integral $\int_{0}^{2 \pi} \cos \tilde{\varphi} g(\sin \tilde{\varphi}) d \tilde{\varphi}=0$. Therefore the current density may be presented in the following form

$\boldsymbol{j}_{\perp}=F\left(B_{0}\right) \boldsymbol{B}_{\perp}$,

where function

$F\left(B_{0}\right)=\sum_{\alpha=i, e} q_{\alpha} B_{0}^{-1} \int v_{\perp}^{2} \sin \tilde{\varphi} f_{\alpha}\left(v_{z}, v_{\perp}, \sin \tilde{\varphi}\right) \mathrm{d} v_{z} \mathrm{~d} v_{\perp} d \tilde{\varphi}$

depends only on the magnetic field magnitude $B_{0}$. The current density determined by Eq. (A1) is in agreement with the magnetic field (1), i.e. $\boldsymbol{j}_{\perp} \times \boldsymbol{B}_{\perp}=0$. Thus, the distribution functions of the form $f_{\alpha}=f_{\alpha}\left(W_{\alpha}, I_{\alpha}\right)$ do allow to develop self-consistent kinetic models of the magnetoplasma structure with the periodic magnetic field (1). 
Acknowledgements. The authors would like to acknowledge Cluster Active Archive and Cluster instrument teams, in particular FGM, CIS, PEACE for excellent data. The work of V.I.Y. was supported by the Russian Foundation for Basic Research (project 13-0200445A). The work of A.V.A. was supported by the Russian Foundation for Basic Research (project 14-02-01269).

Topical Editor L. Blomberg thanks J. De Keyser and one anonymous referee for their help in evaluating this paper.

\section{References}

Artemyev, A. and Zelenyi, L.: Kinetic Structure of Current Sheets in the Earth Magnetotail, Space Sci. Rev., 178, 419-440, doi:10.1007/s11214-012-9954-5, 2013.

Artemyev, A. V.: A model of one-dimensional current sheet with parallel currents and normal component of magnetic field, Phys. Plasmas, 18, 022104, doi:10.1063/1.3552141, 2011.

Artemyev, A. V., Petrukovich, A. A., Zelenyi, L. M., Nakamura, R., Malova, H. V., and Popov, V. Y.: Thin embedded current sheets: Cluster observations of ion kinetic structure and analytical models, Ann. Geophys., 27, 4075-4087, doi:10.5194/angeo27-4075-2009, 2009

Artemyev, A. V., Petrukovich, A. A., Frank, A. G., Nakamura, R., and Zelenyi, L. M.: Intense current sheets in the magnetotail: Peculiarities of electron physics, J. Geophys. Res. Space Phys., 118, 2789-2799, doi:10.1002/jgra.50297, 2013.

Artemyev, A. V., Vasko, I. Y., and Kasahara, S.: Thin current sheets in the Jovian magentotail, Planet Space Sci., 5, 425-437, 2014.

Asano, Y., Nakamura, R., Baumjohann, W., Runov, A., Vörös, Z., Volwerk, M., Zhang, T. L., Balogh, A., Klecker, B., and Rème, H.: How typical are atypical current sheets?, Geophys. Res. Lett., 32, L03108, doi:10.1029/2004GL021834, 2005.

Baker, D. N., Pulkkinen, T. I., Angelopoulos, V., Baumjohann, W., and McPherron, R. L.: Neutral line model of substorms: Past results and present view, J. Geophys. Res., 101, 12975-13010, doi:10.1029/95JA03753, 1996.

Balogh, A., Carr, C. M., Acuña, M. H., Dunlop, M. W., Beek, T. J., Brown, P., Fornacon, K.-H., Georgescu, E., Glassmeier, K.H., Harris, J., Musmann, G., Oddy, T., and Schwingenschuh, K.: The Cluster Magnetic Field Investigation: overview of in-flight performance and initial results, Ann. Geophys., 19, 1207-1217, doi:10.5194/angeo-19-1207-2001, 2001.

Baumjohann, W., Roux, A., Le Contel, O., Nakamura, R., Birn, J., Hoshino, M., Lui, A. T. Y., Owen, C. J., Sauvaud, J.-A., Vaivads, A., Fontaine, D., and Runov, A.: Dynamics of thin current sheets: Cluster observations, Ann. Geophys., 25, 13651389, doi:10.5194/angeo-25-1365-2007, 2007.

Behannon, K. W., Burlaga, L. F., and Ness, N. F.: The Jovian magnetotail and its current sheet, J. Geophys. Res., 86, 8385-8401, doi:10.1029/JA086iA10p08385, 1981.

Bell, T. F.: Nonlinear Alfvén Waves in a Vlasov Plasma, Phys. Fluids, 8, 1829-1839, doi:10.1063/1.1761115, 1965.

Birn, J., Thomsen, M. F., and Hesse, M.: Electron acceleration in the dynamic magnetotail: Test particle orbits in three-dimensional magnetohydrodynamic simulation fields, Phys. Plasmas, 11, 1825-1833, doi:10.1063/1.1704641, 2004.

Birn, J., Artemyev, A. V., Baker, D. N., Echim, M., Hoshino, M., and Zelenyi, L. M.: Particle Acceleration in the Magnetotail and
Aurora, Space Sci. Rev., 173, 49-102, doi:10.1007/s11214-0129874-4, 2012.

Büchner, J. and Zelenyi, L. M.: Regular and chaotic charged particle motion in magnetotaillike field reversals. I - Basic theory of trapped motion, J. Geophys. Res., 94, 11821-11842, doi:10.1029/JA094iA09p11821, 1989.

Chanteur, G.: Spatial Interpolation for Four Spacecraft: Theory, in: Analysis Methods for Multi-Spacecraft Data, edited by: Patschmann, G. and Daly, P. W., 349-370, 2000.

De Keyser, J. and Echim, M.: Electric potential differences across auroral generator interfaces, Ann. Geophys., 31, 251-261, doi:10.5194/angeo-31-251-2013, 2013.

De Keyser, J., Echim, M., and Roth, M.: Cross-field flow and electric potential in a plasma slab, Ann. Geophys., 31, 1297-1314, doi:10.5194/angeo-31-1297-2013, 2013.

Eastwood, J. W.: Consistency of fields and particle motion in the 'Speiser' model of the current sheet, Planet. Space Sci., 20, 1555-1568, doi:10.1016/0032-0633(72)90182-1, 1972.

Eastwood, J. W.: The warm current sheet model, and its implications on the temporal behaviour of the geomagnetic tail, Planet. Space Sci., 22, 1641-1668, doi:10.1016/0032-0633(74)90108-1, 1974.

Fairfield, D. H.: On the average configuration of the geomagnetic tail, J. Geophys. Res., 84, 1950-1958, doi:10.1029/JA084iA05p01950, 1979.

Grigorenko, E. E., Malova, H. V., Artemyev, A. V., Mingalev, O. V., Kronberg, E. A., Koleva, R., Daly, P. W., Cao, J. B., Sauvaud, J.A., Owen, C. J., and Zelenyi, L. M.: Current sheet structure and kinetic properties of plasma flows during a near-Earth magnetic reconnection under the presence of a guide field, J. Geophys.l Res. Space Phys., 118, 3265-3287, doi:10.1002/jgra.50310, 2013.

Harris, E.: On a plasma sheet separating regions of oppositely directed magnetic field, Nuovo Cimento, 23, 115-123, 1962.

Harrison, M. G. and Neukirch, T.: One-Dimensional VlasovMaxwell Equilibrium for the Force-Free Harris Sheet, Phys. Rev Lett., 102, 135003, doi:10.1103/PhysRevLett.102.135003, 2009.

Israelevich, P. L., Ershkovich, A. I., and Oran, R.: Current carriers in the bifurcated tail current sheet: Ions or electrons?, J. Geophys. Res., 113, A04215, doi:10.1029/2007JA012541, 2008.

Johnstone, A. D., Alsop, C., Burge, S., Carter, P. J., Coates, A. J., Coker, A. J., Fazakerley, A. N., Grande, M., Gowen, R. A., Gurgiolo, C., Hancock, B. K., Narheim, B., Preece, A., Sheather, P. H., Winningham, J. D., and Woodliffe, R. D.: Peace: a Plasma Electron and Current Experiment, Space Sci. Rev., 79, 351-398, doi:10.1023/A:1004938001388, 1997.

Lutomirski, R. F. and Sudan, R. N.: Exact Nonlinear Electromagnetic Whistler Modes, Phys. Rev., 147, 156-165, doi:10.1103/PhysRev.147.156, 1966.

McComas, D. J., Bame, S. J., Russell, C. T., and Elphic, R. C.: The near-earth cross-tail current sheet - Detailed ISEE 1 and 2 case studies, J. Geophys. Res., 91, 4287-4301, doi:10.1029/JA091iA04p04287, 1986.

Mingalev, O. V., Mingalev, I. V., Mel'nik, M. N., Artemyev, A. V., Malova, H. V., Popov, V. Y., Chao, S., and Zelenyi, L. M.: Kinetic models of current sheets with a sheared magnetic field, Plasma Phys. Reports, 38, 300-314, doi:10.1134/S1063780X12030063, 2012. 
Nakamura, R., Baumjohann, W., Runov, A., and Asano, Y.: Thin Current Sheets in the Magnetotail Observed by Cluster, Space Sci. Rev., 122, 29-38, doi:10.1007/s11214-006-6219-1, 2006.

Nakamura, R., Baumjohann, W., Fujimoto, M., Asano, Y., Runov, A., Owen, C. J., Fazakerley, A. N., Klecker, B., RèMe, H., Lucek, E. A., Andre, M., and Khotyaintsev, Y.: Cluster observations of an ion-scale current sheet in the magnetotail under the presence of a guide field, J. Geophys. Res. Space Phys., 113, A07S16, doi:10.1029/2007JA012760, 2008.

Ness, N. F.: The Earth's Magnetic Tail, J. Geophys. Res., 70, 29893005, doi:10.1029/JZ070i013p02989, 1965.

Ness, N. F.: The geomagnetic tail., Rev. Geophys. Space Phys., 7, 97-127, doi:10.1029/RG007i001p00097, 1969.

Nickeler, D. H. and Wiegelmann, T.: Thin current sheets caused by plasma flow gradients in space and astrophysical plasma, Ann. Geophys., 28, 1523-1532, doi:10.5194/angeo-28-15232010, 2010.

Panov, E. V., Artemyev, A. V., Nakamura, R., and Baumjohann, W.: Two types of tangential magnetopause current sheets: Cluster observations and theory, J. Geophys. Res., 116, A12204, doi:10.1029/2011JA01686, 2011.

Petrukovich, A. A.: Origins of plasma sheet $B_{y}$, J. Geophys. Res., 116, A07217, doi:10.1029/2010JA016386, 2011.

Petrukovich, A. A., Artemyev, A. V., Malova, H. V., Popov, V. Y., Nakamura, R., and Zelenyi, L. M.: Embedded current sheets in the Earth magnetotail, J. Geophys. Res. Space Phys., 116, A00I25, doi:10.1029/2010JA015749, 2011.

R`eme, H., Aoustin, C., Bosqued, J. M., Dandouras, I., Lavraud, B., Sauvaud, J. A., Barthe, A., Bouyssou, J., Camus, Th., CoeurJoly, O., Cros, A., Cuvilo, J., Ducay, F., Garbarowitz, Y., Medale, J. L., Penou, E., Perrier, H., Romefort, D., Rouzaud, J., Vallat, C., Alcaydé, D., Jacquey, C., Mazelle, C., d'Uston, C., Möbius, E., Kistler, L. M., Crocker, K., Granoff, M., Mouikis, C., Popecki, M., Vosbury, M., Klecker, B., Hovestadt, D., Kucharek, H., Kuenneth, E., Paschmann, G., Scholer, M., Sckopke, N., Seidenschwang, E., Carlson, C. W., Curtis, D. W., Ingraham, C., Lin, R. P., McFadden, J. P., Parks, G. K., Phan, T., Formisano, V., Amata, E., Bavassano-Cattaneo, M. B., Baldetti, P., Bruno, R., Chionchio, G., Di Lellis, A., Marcucci, M. F., Pallocchia, G., Korth, A., Daly, P. W., Graeve, B., Rosenbauer, H., Vasyliunas, V., McCarthy, M., Wilber, M., Eliasson, L., Lundin, R., Olsen, S., Shelley, E. G., Fuselier, S., Ghielmetti, A. G., Lennartsson, W., Escoubet, C. P., Balsiger, H., Friedel, R., Cao, J.-B., Kovrazhkin, R. A., Papamastorakis, I., Pellat, R., Scudder, J., and Sonnerup, B.: First multispacecraft ion measurements in and near the Earth's magnetosphere with the identical Cluster ion spectrometry (CIS) experiment, Ann. Geophys., 19, 1303-1354, doi:10.5194/angeo19-1303-2001, 2001.

Rong, Z. J., Wan, W. X., Shen, C., Li, X., Dunlop, M. W., Petrukovich, A. A., Hau, L.-N., Zhang, T. L., Rème, H., Du, A. M., and Lucek, E.: Profile of strong magnetic field $\mathrm{B}_{y}$ component in magnetotail current sheets, J. Geophys. Res. Space Phys., 117, A06216, doi:10.1029/2011JA017402, 2012.

Roth, M., De Keyser, J., and Kuznetsova, M. M.: Vlasov Theory of the Equilibrium Structure of Tangential Discontinuities in Space Plasmas, Space Sci. Rev., 76, 251-317, doi:10.1007/BF00197842, 1996.

Runov, A., Sergeev, V. A., Nakamura, R., Baumjohann, W., Apatenkov, S., Asano, Y., Takada, T., Volwerk, M., Vörös, Z.,
Zhang, T. L., Sauvaud, J.-A., Rème, H., and Balogh, A.: Local structure of the magnetotail current sheet: 2001 Cluster observations, Ann. Geophys., 24, 247-262, doi:10.5194/angeo-24-2472006, 2006.

Schindler, K., Birn, J., and Hesse, M.: Kinetic model of electric potentials in localized collisionless plasma structures under steady quasi-gyrotropic conditions, Phys. Plasmas, 19, 082904 , doi:10.1063/1.4747162, 2012.

Sergeev, V. A., Mitchell, D. G., Russell, C. T., and Williams, D. J.: Structure of the tail plasma/current sheet at $\sim 11 R_{E}$ and its changes in the course of a substorm, J. Geophys. Res., 98, 17345-17366, doi:10.1029/93JA01151, 1993.

Sergeev, V. A., Angelopoulos, V., and Nakamura, R.: Recent advances in understanding substorm dynamics, Geophys. Res. Lett., 39, L05101, doi:10.1029/2012GL050859, 2012.

Shen, C., Liu, Z. X., Li, X., Dunlop, M., Lucek, E., Rong, Z. J., Chen, Z. Q., Escoubet, C. P., Malova, H. V., Lui, A. T. Y., Fazakerley, A., Walsh, A. P., and Mouikis, C.: Flattened current sheet and its evolution in substorms, J. Geophys. Res. Space Phys., 113, A07S21, doi:10.1029/2007JA012812, 2008a.

hen, C., Rong, Z. J., Li, X., Dunlop, M., Liu, Z. X., Malova, H. V., Lucek, E., and Carr, C.: Magnetic configurations of the tilted current sheets in magnetotail, Ann. Geophys., 26, 3525-3543, doi:10.5194/angeo-26-3525-2008, 2008b.

Sitnov, M. I., Zelenyi, L. M., Malova, H. V., and Sharma, A. S.: Thin current sheet embedded within a thicker plasma sheet: Selfconsistent kinetic theory, J. Geophys. Res., 105, 13029-13044, doi:10.1029/1999JA000431, 2000.

Sonnerup, B. U. Ö. and Cahill Jr., L. J.: Explorer 12 observations of the magnetopause current layer, J. Geophys. Res., 73, 1757, doi:10.1029/JA073i005p01757, 1968.

Sonnerup, B. U. Ö. and Su, S.-Y.: Large Amplitude Whistler Waves in a Hot Collision-Free Plasma, Phys. Fluids, 10, 462-464, doi:10.1063/1.1762132, 1967.

Speiser, T. W.: Particle Trajectories in Model Current Sheets, 1, Analytical Solutions, J. Geophys. Res., 70, 4219-4226, doi:10.1029/JZ070i017p04219, 1965.

Vasko, I. Y., Artemyev, A. V., Popov, V. Y., and Malova, H. V.: Kinetic models of two-dimensional plane and axially symmetric current sheets: Group theory approach, Phys. Plasmas, 20, 022110, doi:10.1063/1.4792263, 2013.

Vasko, I. Y., Artemyev, A. V., and Zelenyi, L. M.: Large-amplitude circularly polarized electromagnetic waves in magnetized plasma, Phys. Plasmas, 21, 054501, doi:10.1063/1.4875339, 2014.

Yoon, P. H. and Lui, A. T. Y.: Model of ion- or electrondominated current sheet, J. Geophys. Res., 109, A11213, doi:10.1029/2004JA010555, 2004.

Yoon, P. H. and Lui, A. T. Y.: A class of exact two-dimensional kinetic current sheet equilibria, J. Geophys. Res., 110, A01202, doi:10.1029/2003JA010308, 2005.

Zelenyi, L. M., Sitnov, M. I., Malova, H. V., and Sharma, A. S.: Thin and superthin ion current sheets. Quasi-adiabatic and nonadiabatic models, Nonlin. Processes Geophys., 7, 127-139, doi:10.5194/npg-7-127-2000, 2000.

Zelenyi, L. M., Artemyev, A. V., and Petrukovich, A. A.: Earthward electric field in the magnetotail: Cluster observations and theoretical estimates, Geophys. Res. Lett., 37, L17110, doi:10.1029/2009GL042099, 2010. 